\title{
Preventive Care
}

\section{Is Some Provider Advice on Smoking Cessation Better Than No Advice? An Instrumental Variable Analysis of the 2001 National Health Interview Survey}

\author{
Yuhua Bao, Naihua Duan, and Sarah A. Fox
}

Research Objective. To estimate the effect of provider advice in routine clinical contacts on patient smoking cessation outcome.

Data Source. The Sample Adult File from the 2001 National Health Interview Survey. We focus on adult patients who were either current smokers or quit during the last 12 months and had some contact with the health care providers or facilities they most often went to for acute or preventive care.

Study Design. We estimate a joint model of self-reported smoking cessation and ever receiving advice to quit during medical visits in the past 12 months. Because providers are more likely to advise heavier smokers and/or patients already diagnosed with smoking-related conditions, we use provider advice for diet/nutrition and for physical activity reported by the same patient as instrumental variables for smoking cessation advice to mitigate the selection bias. We conduct additional analyses to examine the robustness of our estimate against the various scenarios by which the exclusion restriction of the instrumental variables may fail.

Principal Findings. Provider advice doubles the chances of success in (self-reported) smoking cessation by their patients. The probability of quitting by the end of the 12 month reference period increased from 6.9 to 14.7 percent, an effect that is of both statistical $(p<.001)$ and clinical significance.

Conclusions. Provider advice delivered in routine practice settings has a substantial effect on the success rate of smoking cessation among smoking patients. Providing advice consistently to all smoking patients, compared with routine care, is more effective than doubling the federal excise tax and, in the longer run, likely to outperform some of the other tobacco control policies such as banning smoking in private workplaces.

Key Words. Smoking cessation, provider advice, instrumental variables, exclusion restriction, bivariate probit 
Despite much increased awareness of the health hazards of smoking, 23 percent of American adult population remain smokers in 2001 (National Center for Health Statistics 2003a). Although the majority (about two-thirds) of smoking adults had some contact with health care providers annually, physicians were advising patients to quit in only one out of five encounters with smokers (Thorndike et al. 1998), suggesting missed opportunities of provider intervention. What adds to the concern, other than the low rate of advice, is that health care providers are more likely to advise patients who are heavier smokers and/or who already have tobacco-related conditions such as heart disease, cancer, and chronic obstructive pulmonary diseases (COPD) (e.g., Fiore et al. 1990; Gilpin et al. 1993; Jaén et al. 1997). By doing so, providers largely miss out on the opportunities of converting lighter smokers for whom quitting might be easier, and of preventing the damages of smoking. Among a number of reasons for which providers fail to provide advice during clinical encounters with smoking patients, the lack of confidence in the effectiveness of their advice has proven to be a great barrier (Demers et al. 1990).

This study aims to estimate the effectiveness of provider advice on smoking cessation in the real world under time and other resource constraints and in an unselected patient population. A number of studies based on randomized controlled trials (RCTs) have tested the efficacy of smoking cessation advice delivered by health care providers (e.g., Kottke et al. 1988; Fiore et al. 2000), usually under strictly controlled conditions. The lack of flexibility in the treatment conditions thus makes it hard to generalize the evidence from RCTs to the experience of community practitioners who develop intervention strategies to suit their own practice styles and the needs of their own patients. In addition, clinical trials often adopt restrictive criteria when recruiting participating patients and providers and are usually conducted in nonrepresentative clinical settings such as academic medical centers. Therefore, intervention

Address correspondence to Yuhua Bao, Ph.D., Center for Community Partnerships in Health Promotion, Division of General Internal Medicine and Health Services Research, David Geffen School of Medicine at UCLA, 1100 Glendon Ave, Ste 2010, Los Angeles, CA 90024-3524. Naihua Duan, Ph.D., is with the Center for Community Health, Jane \& Terry Semel Institute for Neuroscience and Human Behavior, UCLA, Los Angeles, CA. Dr. Duan is also with Department of Psychiatry and Biobehavioral Sciences, David Geffen School of Medicine at UCLA, Los Angeles, CA and with the Department of Biostatistics, School of Public Health, Los Angeles, CA 900246543. Sarah A. Fox, Ed.D., M.S.P.H., is with the Center for Community Partnerships in Health Promotion, Division of General Internal Medicine and Health Services Research, David Geffen School of Medicine at UCLA, Los Angeles, CA 90024-3524. 
outcomes as seen in RCTs might not be replicable among the patient population in community settings.

Furthermore, what RCTs are designed to identify, namely, the average efficacy of a well-defined intervention across a specific patient population, is usually not adequate to inform the decisions of providers in busy community practices. As providers have to choose between to advise and not to advise when the next smoking patient steps into their office, they could be better informed by knowing how effective it would be if they increase their advice incrementally.

To inform policy and practice, this study estimates the effect of health provider advice, delivered in routine care settings, on the success of smoking cessation by their patients, using nationally representative survey data of patients receiving care from their providers. To address the selection in provider advice of smoking cessation in day-to-day practice-for example, heavier smokers and/or patients with smoking-related conditions are more likely to be advised-we use the behavior of the same provider in advising diet and physical activities as instrumental variables (IVs) for smoking cessation advice (see, e.g., McClellan and Newhouse [2000] for an overview of the application of the IV method in health services research and the other articles in the same special issue of Health Services Research 35[5]). We model smoking cessation outcome and provider advice jointly. The effect of "some advice" (versus no advice) is predicted based on the model, and the robustness of the effect size against the crucial assumption for the identification is examined by additional analyses including a simulation exercise.

\section{DATA AND METHODS}

\section{Study Sample}

This study uses data from the 2001 National Health Interview Survey (NHIS) Sample Adult File. NHIS is an annual household survey on various health topics and represents the civilian, noninstitutionalized U.S. population $(\mathrm{Na}-$ tional Center for Health Statistics 2003b). Of the 33,326 persons interviewed for the sample adult questions, 7,662 were self-reported current smokers, and 712 reported that they quit smoking within the last 12 months. Of the 8,374 current smokers and recent quitters, 5,512 had been in contact with their regular health providers or health facilities during the last 12 months, and therefore were asked whether their providers advised them to quit smoking. The present study focuses on this subsample of individuals $(n=5,512)$. We 
provide in Table 1 definitions and summary statistics of key variables on patient sociodemographics, health conditions, and smoking-related information for the entire analysis sample.

\section{Definition of Smoking Cessation}

Smoking cessation within the last 12 months is determined if a person who ever smoked regularly (defined as ever smoked 100 cigarettes in one's entire life) reported "not smoking at all" (versus everyday or some days) currently and reported quitting within the last 12 months. Previous studies have found self-report data to be fairly reliable and accurate measures of smoking status compared with biochemical assessments (Patrick et al. 1994; Pokorski, Chen, and Bertholf 1994; Assaf et al. 2002). For example, Patrick et al. (1994), a meta-analysis of the validity of self-reported smoking, found the sensitivity of self-reports to be 87.5 percent and the specificity 89.2 percent.

\section{Definition of Provider Advice}

Provider advice to quit smoking was defined by a positive answer to the question:

"During the past 12 months, has your-(provider most often seen or health facility usually went to for acute or preventive care) advised you to quit smoking?"

Provider advice for diet/nutrition and for physical activity were determined by two similar questions for persons who had been in contact with their usual sources of care in the last 12 months:

"During the past 12 months, has your-(provider most often seen or health facility usually went to for acute or preventive care) asked you about or given you advice regarding

... diet and nutrition?

... physical activity or exercise?"

\section{Providers Select to Advise Certain Patients}

That providers advise smoking cessation in a nonrandom manner is illustrated in Table 2, where we show the weighted means of tobacco consumption indicators and prevalence of smoking-related health conditions by whether or not the patient received advice during the 12-month reference period. Summary statistics indicate that patients who received some advice on average had a longer history of regular smoking than patients who did not receive any 
Table 1: Summary Statistics of Selected Patient Information of the Analysis Sample

\begin{tabular}{|c|c|}
\hline & Entire Sample \\
\hline \multicolumn{2}{|l|}{ Demographics } \\
\hline Age at time of the interview & $42.73(15.34)[18,85]$ \\
\hline Female & $54.7 \%$ \\
\hline White & $80.0 \%$ \\
\hline Black & $11.1 \%$ \\
\hline Hispanic & $6.0 \%$ \\
\hline Other race & $2.8 \%$ \\
\hline Living with spouse or partner & $59.9 \%$ \\
\hline \multicolumn{2}{|l|}{ Education } \\
\hline Less than high school & $18.6 \%$ \\
\hline High school graduates & $35.0 \%$ \\
\hline Some college & $23.7 \%$ \\
\hline College or above & $22.7 \%$ \\
\hline \multicolumn{2}{|l|}{ Health condition } \\
\hline Ever had serious cardiovascular conditions ${ }^{\dagger}$ & $9.3 \%$ \\
\hline Ever had other heart conditions $s^{\ddagger}$ & $29.9 \%$ \\
\hline Lung/breathing-related conditions $\mathrm{s}^{\S}$ & $22.1 \%$ \\
\hline Ever diagnosed with cancer & $8.0 \%$ \\
\hline Reported worse health than a year ago & $13.7 \%$ \\
\hline \multicolumn{2}{|l|}{ Smoking-related information } \\
\hline Quit smoking during the last 12 months & $10.1 \%$ \\
\hline Years of regular smoking & $25.17(15.26)[0,77]$ \\
\hline Age first started to smoke regularly: 15 or younger & $32.0 \%$ \\
\hline Age first started to smoke regularly: $16-20$ & $51.0 \%$ \\
\hline Age first started to smoke regularly: 21 or older & $16.9 \%$ \\
\hline \multicolumn{2}{|l|}{ Health insurance coverage } \\
\hline Uninsured & $12.4 \%$ \\
\hline Medicaid but not Medicare & $7.4 \%$ \\
\hline Medicare but not Medicaid & $10.3 \%$ \\
\hline Medicare and Medicaid dual coverage & $2.8 \%$ \\
\hline Private insurance only & $63.5 \%$ \\
\hline Other public insurance & $3.6 \%$ \\
\hline \multicolumn{2}{|l|}{ Other } \\
\hline Working now & $65.7 \%$ \\
\hline Index of social support* & $0.70(0.23)[0,1]$ \\
\hline \multicolumn{2}{|l|}{ Provider advised to quit, 12 months } \\
\hline Sample size & 5,512 \\
\hline
\end{tabular}

Notes: Authors' calculation using the NHIS 2001 sample adult file; all statistics are weighted to be nationally representative; standard deviation and range for continuous variables are reported in parentheses and square brackets, respectively.

*The index of social support is constructed by taking the average of six dichotomous indicators of social contacts in the last 2 weeks: getting together socially with friends or neighbors; talking with friends or neighbors on the telephone; getting together with any relatives not including those living with you; talking with any relatives on the telephone not including those living with you; going to a church, temple, or another place of worship for services or other activities; going to a show or movie, sports event, club meeting, class, or other group event.

'Including myocardial infarction, coronary heart diseases, angina, and stroke.

${ }^{\ddagger}$ Including hypertension and other heart conditions.

${ }^{\S}$ Including ever had emphysema or asthma, or diagnosed with chronic bronchitis in the last 12 months. 


\section{Table 2: Smoking-Related Information by Whether Providers Advised to} Quit in the Past 12 Months

\begin{tabular}{|c|c|c|c|}
\hline Smoking History and Current Consumption & $\begin{array}{l}\text { Provider Did Not } \\
\text { Advise to Quit, } \\
12 \text { Months }\end{array}$ & $\begin{array}{l}\text { Provider Advised } \\
\text { to Quit, } 12 \text { Months }\end{array}$ & $p$-Value \\
\hline \multicolumn{4}{|l|}{ Current smokers and recent quitters (entire sample) } \\
\hline Quit smoking, 12 months & $16.0 \%$ & $7.0 \%$ & $<.001$ \\
\hline Years of regular smoking* & $\begin{array}{l}23.09 \\
(15.72)[0,75]\end{array}$ & $\begin{array}{l}26.20 \\
(14.92)[0,77]\end{array}$ & .099 \\
\hline \multicolumn{4}{|l|}{ Age first started to smoke regularly: } \\
\hline 15 or younger & $29.7 \%$ & $33.2 \%$ & .472 \\
\hline Age first started to smoke regularly: $16-20$ & $51.3 \%$ & $50.9 \%$ & .887 \\
\hline Age first started to smoke regularly: 21 or older & $19.0 \%$ & $15.9 \%$ & .195 \\
\hline \multicolumn{4}{|l|}{ Current smokers only } \\
\hline Everday (versus some-day) smokers & $71.0 \%$ & $87.0 \%$ & .025 \\
\hline Number of cigarettes smoked per day & $\begin{array}{l}13.09 \\
(10.39)[1,60]\end{array}$ & $\begin{array}{l}16.43 \\
(10.33)[1,90]\end{array}$ & .048 \\
\hline $\begin{array}{l}\text { Attempted quitting by stopping smoking for } \\
\text { at least one day, } 12 \text { months }\end{array}$ & $35.8 \%$ & $49.1 \%$ & .006 \\
\hline \multicolumn{4}{|l|}{ Smoking-related conditions (entire sample) } \\
\hline Ever had serious cardiovascular conditions ${ }^{\dagger}$ & $7.0 \%$ & $12.0 \%$ & .004 \\
\hline Ever had other heart conditions ${ }^{\ddagger}$ & $26.3 \%$ & $35.0 \%$ & .137 \\
\hline Lung/breathing-related conditions ${ }^{\S}$ & $17.8 \%$ & $26.1 \%$ & .033 \\
\hline Ever diagnosed with cancer & $6.6 \%$ & $9.5 \%$ & .006 \\
\hline \multicolumn{4}{|l|}{$\begin{array}{l}\text { Provider advice for other health } \\
\text { behaviors (entire sample) }\end{array}$} \\
\hline Provider advised diet/nutrition & $26.8 \%$ & $49.3 \%$ & 0.086 \\
\hline Provider advised physical activity & $29.2 \%$ & $49.5 \%$ & 0.001 \\
\hline $\begin{array}{l}\text { Predicted probability of quitting based } \\
\text { on a probit model } \\
\text { (not correcting for selection bias) }\end{array}$ & $15.6 \%$ & $6.9 \%$ & $<.001$ \\
\hline
\end{tabular}

Notes: Authors' calculation using the NHIS 2001 sample adult file; all statistics are weighted to be nationally representative; standard deviations and ranges for continuous variables are reported in parentheses and square brackets, respectively.

$p$-values presented in the last column apply to the differences between the advised group and the non-advised group, adjusting for sample design of NHIS.

Of the entire sample (current smokers and recent quitters), $n=1,916$ for those not advised to quit, and $n=3,596$ for those advised to quite;

Of current smokers, $n=1,624$ for those not advised, and $n=3,351$ for those advised.

*Years of regular smoking is imputed by taking the difference between the respondent's age and the reported age when the respondent "started to smoke fairly regularly."

"Including myocardial infarction, coronary heart diseases, angina, and stroke.

${ }^{\ddagger}$ Including hypertension and other heart conditions.

${ }^{\S}$ Including ever had emphysema or asthma, or diagnosed with chronic bronchitis in the last 12 months. 
advice $(p=.099)$. Of those still smoking at the time of the interview, patients who were advised were more likely to be "every day" (versus "some days") smokers $(p=.025)$, and had higher daily consumption of cigarettes ( $p=.048)$. Furthermore, the data show much higher prevalence of smoking-related health conditions among the advised group than the nonadvised group $(p<.05$ for serious cardiovascular conditions, lung-breathing-related conditions, and cancer). These data suggest that, with limited time and resources, providers tend to advise patients believed to need help the most- - those who are heavier smokers and/or who already have smoking-related health conditions.

This pattern of provider behavior leads to biases in the estimated effect of provider advice in both directions if we simply compare the quitting rate between the advised and nonadvised groups. On the one hand, providers' tendency to advise heavier and longer-term smokers leads to a downward bias in the estimation because heavier smokers generally have a harder time quitting; on the other, patients diagnosed with smoking-related conditions may become more motivated to quit, thus making advice look more effective than it would have been had the advice been delivered to all smoking patients.

It is thus not surprising that although current smoking patients with some advice were more likely to have attempted quitting than the nonadvised smokers (49 versus 36 percent, $p=.006$ ), the rate of success (among current smokers and recent quitters) is less than half among the advised than among the nonadvised ( 7 versus 16 percent, $p<.001$ ). Even after controlling for years of regular smoking, presence of smoking-related conditions and other important covariates as listed in Table 1, results of a probit analysis still suggest a counter-intuitive negative effect of provider advice on cessation $(-8.7$ percentage points, shown in the last row of Table 2). Substantial unobserved differences exist between the advised and the nonadvised groups that are not controllable by the multivariate analysis.

\section{Statistical Strategy}

We use the IV method to statistically correct for the selection bias in provider advice for smoking cessation. The idea of the IV method is to find one or more variables (the "IVs") that are substantially correlated with the treatment variable, but have no direct correlation with the outcome variable of interest. It then uses the variation in the treatment variable that is induced by the $\operatorname{IV}(\mathrm{s})$ (thus considered exogenous to the outcome) to estimate the effect of the treatment. In practical applications, the IV method is often applied using a two-stage procedure: in the first stage, treatment is predicted as a function of 
the IV $(\mathrm{s})$ and other covariates; in the second, the outcome of interest is estimated as a function of the predicted treatment variable and other specified covariates (Angrist, Imbens, and Rubin 1996; Greene 2000).

In our application, we exploit the behavioral pattern of providers in providing preventive care to correct for the underlying selection bias. More specifically, providers who advise on one type of health behavior of the patient (e.g., diet or physical activity) tend to advise on other types (e.g., smoking cessation) as well. Thus we use providers' advice on diet and physical activity as IVs for their smoking cessation advice for the same patient. We make the assumption that provider advice for diet/nutrition and for physical activity are not directly correlated with the patient's likelihood of success in smoking cessation (except through increased likelihood of provider advice for smoking cessation).

The assumption that the IVs are not directly correlated with smoking cessation cannot be tested directly. ${ }^{1}$ But it is worthwhile to discuss the ways by which the IVs may fail the assumption. First of all, the exclusion restriction may be called into question if providers advised on diet or physical activity to address patients' concern about weight gain as a result of quitting. Although this is arguably possible in some cases (e.g., if patients raise the concern when discussing smoking cessation with their providers), weight gain after quitting is quite limited in most cases and providers are much more likely to give such advice in response to patients' weight gain after they quit rather than before (Fiore et al. 2000). Second, our IVs could fail if patients who are more healthminded choose to enroll in health plans or select to see physicians that place greater emphasis on health promotion and prevention, but because of their own health-mindedness, are at the same time more likely to quit smoking. Third, there might be unobserved health conditions for which smoking and/or diet/ physical activity are potential risk factors. This in turn may bias the estimated effect of advice for smoking cessation if patients advised on diet/physical activity because of (unobserved) health conditions are motivated to quit to a different extent than patients without such conditions. ${ }^{2,3}$ Similar concerns arise if unobserved severity of observed conditions play a role in both physician advice and patient quitting outcome. Fourth, there are other issues that might render the exclusion restriction inappropriate including having an unobserved family history of diseases or some family health event (e.g., parental mortality because of cancer or cardiovascular conditions), which makes it more likely for the patient to receive physician advice but also independently motivates the smoker to quit. We conduct additional analyses to address each of these possibilities and report these results in the Results section. 


\section{Model Specification and Predictions}

The two-stage approach to IV estimation, either the two-stage least-squares linear probability model or the two-step probit model, is known to yield inconsistent estimates when both the outcome variable and the endogenous treatment variable are dichotomous (Bhattacharya Goldman, and McCaffrey 2006). Results of a Monte Carlo exercise conducted by Bhattacharya and colleagues argued in favor of the multivariate probit model compared with the two-stage least squares or the two-step probit estimator. This is especially true when there are multiple (endogenous) treatments, when the average probability of the dependent variable is close to zero or one, and when the data generating process is not normal. Given the low cessation rate in our sample (around 10 percent), we use the bivariate probit model to jointly model smoking cessation outcome and provider advice, explicitly taking into account the correlation between the unobserved factors in providers' advice to quit smoking and those in patients' success of smoking cessation. To check the robustness of our results with regard to different estimators, we also fit a two-stage least-squares model (Angrist 2001) and a two-step probit model to the data.

We include the following variables in the equation for "some advice" versus "no advice": the two IVs, patient demographics (age groups, omitting 18-34 as the reference group; racial/ethnic groups, omitting "white" as the reference group; gender, omitting "male" as the reference group), education (less than high school, high school, some college, omitting college or higher education as the reference), a dummy indicator of living with one's spouse or partner, an indicator of working now, the index of social support, age at which the patient started smoking regularly (15 or earlier, $16-20$, and 21 or later, with the most populous group, 16-20, omitted as the reference), the four indicators of smoking-related conditions, and a set of insurance variables (uninsured, Medicaid but not Medicare, Medicare but not Medicaid, Medicaid and Medicare dual coverage, private insurance only and other public coverage, with the uninsured being the reference group). In the equation for the smoking cessation outcome, in addition to the treatment variable of some provider advice to quit smoking, we include the same set of covariates except the two IVs. ${ }^{4}$ In a separate analysis, instead of the one indicator of "any lung/breathing condition," we use three dummy variables for emphysema, chronic bronchitis, and asthma. Likelihood-ratio test suggests no significant difference between these two specifications.

To facilitate interpretation of estimated coefficients of the bivariate probit model, we derive incremental probability of receiving the advice or of 
quitting associated with each variable in the model (see notes of Table 3 for how the incremental probabilities are derived for dichotomous, categorical, and continuous variables). By applying analysis weight to the calculation of incremental probabilities, we take into account the complex sample design of the NHIS.

\section{RESULTS}

\section{Main Results}

Table 3 shows the output of the bivariate probit model. In the advice equation, both IVs turned out to be strong and highly significant predictors of provider advice on smoking cessation $(p<.001)$. The incremental probabilities of smoking cessation advice associated with advice on physical activity and on diet were 9.7 and 13.7 percentage points, respectively. Older patients were more likely to receive advice from their providers, except that the pattern reversed among the $65+$ group, possibly due to the fact that more acute and chronic health problems of the elderly patients dominated the need of preventive care counseling. Female (versus male) patients were more likely $(p=.005)$, and Hispanic (versus white) patients less likely $(p<.001)$, to be advised. Starting smoking at an older age, compared with the modal age group of 16-20, was associated with lower chances of being advised $(p<.001)$. Ever having serious cardiovascular conditions and the presence of lung/breathingrelated conditions are both strong predictors of providers' advice for smoking cessation ( $p=.007$ and $p<.001$, respectively). Having less serious heart conditions and ever being diagnosed with cancer also positively predicted advice, although not statistically significantly.

The estimated coefficient of advice in the outcome equation suggests that provider advice significantly increased the probability that the patient had quit during the past 12 months $(p=.001)$. The effect of some provider advice to quit smoking amounted to more than doubling the probability of quitting in the last 12 months from 6.9 percent (bootstrap standard error $=0.7$ percent) to 14.7 percent (bootstrap standard error $=1.7$ percent), indicating an effect of a 7.8 percentage point $(\mathrm{SE}=2.2)$ increase in the probability of quitting attributable to provider advice. Compared with younger patients, both the mid-age (35-49 year olds) and near-elderly (50-64 year olds) patients were less likely to have quit. Elderly $(65+)$ patients, on the other hand, were not significantly different from patients younger than 35 . This is probably because those who did not quit at a younger age were heavier and longer-term smokers. Female 
Table 3: Estimated Bivariate Probit Model of Provider Advice and Quit Outcome

\begin{tabular}{|c|c|c|c|c|c|c|}
\hline & \multicolumn{3}{|c|}{ Advise Equation } & \multicolumn{3}{|c|}{ Quit Equation } \\
\hline & Coefficient & $p$-Value & $\begin{array}{l}\text { Incremental } \\
\text { Probability* }\end{array}$ & Coefficient & $p$-Value & $\begin{array}{l}\text { Incremental } \\
\text { Probability* }\end{array}$ \\
\hline $\begin{array}{l}\text { Advised by provider to quit } \\
\text { smoking, } 12 \text { months }\end{array}$ & & & & 0.444 & .001 & .078 \\
\hline $\begin{array}{l}\text { Provider advised on physical } \\
\text { activity, } 12 \text { months }\end{array}$ & 0.281 & .000 & .097 & & & \\
\hline $\begin{array}{l}\text { Provider advised on diet/ } \\
\text { nutrition, } 12 \text { months }\end{array}$ & 0.398 & .000 & .137 & & & \\
\hline Patient age $35-49$ & 0.195 & .000 & .068 & -0.325 & .000 & -.066 \\
\hline Patient age $50-64$ & 0.293 & .000 & .101 & -0.365 & .000 & -.072 \\
\hline Patient age $65+$ & 0.208 & .035 & .073 & -0.111 & .351 & -.025 \\
\hline Patient is female & 0.107 & .005 & .037 & -0.136 & .004 & -.026 \\
\hline Patient is black & -0.095 & .089 & -.033 & -0.005 & .945 & -.001 \\
\hline Patient is Hispanic & -0.345 & .000 & -.123 & 0.111 & .163 & .023 \\
\hline Patient is of other race/ethnicity & 0.052 & .662 & .017 & -0.121 & .428 & -.022 \\
\hline Less than high school education & 0.064 & .292 & .022 & -0.264 & .001 & -.052 \\
\hline High school education & 0.103 & .039 & .035 & -0.197 & .001 & -.040 \\
\hline Some college & 0.073 & .171 & .025 & -0.109 & .091 & -.023 \\
\hline Living with spouse or partner & 0.053 & .164 & .018 & 0.036 & .447 & .007 \\
\hline Working now & 0.098 & .039 & .034 & -0.108 & .066 & -.021 \\
\hline Index of social support & 0.153 & .066 & .012 & 0.146 & .180 & .007 \\
\hline $\begin{array}{l}\text { Patient age when started } \\
\text { smoking: } 15 \text { or earlier }\end{array}$ & 0.035 & .417 & .012 & 0.076 & .157 & .015 \\
\hline $\begin{array}{l}\text { Patient age when started } \\
\text { smoking: } 21 \text { or later }\end{array}$ & -0.223 & .000 & -.078 & 0.007 & .909 & .001 \\
\hline $\begin{array}{l}\text { Ever had serious cardiovascular } \\
\text { conditions }\end{array}$ & 0.191 & .007 & .063 & 0.072 & .390 & .014 \\
\hline Ever had other heart condition & 0.065 & .145 & .022 & 0.001 & .989 & .000 \\
\hline Lung/breathing- related conditions & 0.241 & .000 & .080 & -0.028 & .624 & -.005 \\
\hline Ever diagnosed with cancer & 0.109 & .120 & .037 & -0.027 & .756 & -.005 \\
\hline Medicaid but not Medicare & 0.150 & .068 & .050 & -0.029 & .786 & -.005 \\
\hline Medicare but not Medicaid & 0.030 & .768 & .010 & 0.015 & .903 & .003 \\
\hline $\begin{array}{l}\text { Dually covered by Medicaid } \\
\text { and Medicare }\end{array}$ & -0.040 & .739 & -.014 & 0.310 & .030 & .066 \\
\hline Private insurance only & -0.005 & .924 & -.002 & 0.088 & .245 & .016 \\
\hline Other public insurance & 0.176 & .110 & .059 & 0.245 & .058 & .050 \\
\hline Constant & -0.379 & .000 & & -1.238 & .000 & \\
\hline$\rho$ & -0.570 & .000 & & & & \\
\hline
\end{tabular}

Notes: $n=5,320$.

*For dichotomous variables, incremental probability is derived by switching the variable from 0 to 1 for each individual and taking the weighted mean difference between the two probabilities; for categorical variables (age, race/ethnicity, age when started smoking), incremental probability is derived in a similar way, with the other nonomitted categories set to 0 ; for the continuous variable "index of social support," incremental probability is the difference between the probability when social support takes its sample mean $(0.70)$ and when it is one standard deviation (0.23) above the mean. 
patients were less likely to have quit relative to males. Lower education was associated with lower, and living with spouse or partner with greater, likelihood of quitting. The index of social support, as expected, was positively correlated with quitting, yet not statistically significant. Starting smoking in one's early teens was associated with greater, and starting in one's 20 s or later was associated lower, chances of quitting, compared with starting between the age of 16 and 20 .

The correlation between the error terms of the two probit equations was negative and statistically significant $(\rho=-0.57, p<.001)$, suggesting that providers tended to advise patients who had lower chances of quitting, and the covariates included in the model could not entirely account for the selection.

The two-step probit model resulted in an even larger estimate for the effect of provider advice: an increase in the quitting rate from 3.6 to 15.6 percent $(p<.001)$. The corresponding two-stage least-squares model predicted quitting rate in absence of a provider's advice at 0.6 percent and the effect of provider advice was estimated to be 14.2 percentage points $(p<.001)$. The extremely low rate without the advice was likely due to the extrapolation toward zero by the linear probability model, which tends to break down for small probabilities.

\section{Analyses Regarding the Identification Assumption}

We conducted several analyses to check the possibilities by which our identification assumption - the IVs do not directly affect a patient's chances of quitting - may fail, as discussed in Methods. We first restricted the analysis sample to the privately insured $(n=3,097)^{5}$ and added the indicator of $\mathrm{HMO} /$ IPA (versus other private insurance, including PPO, POS, Fee-for-service/ indemnity, and other) as an additional predictor to both equations. This strategy follows from the notion that health-minded patients are more likely to choose managed care to take advantage of its emphasis on preventive services including lifestyle and behavior counseling. The estimated coefficients of $\mathrm{HMO} / \mathrm{IPA}$ (versus other private insurance) were very small compared with those of other covariates in the model and statistically insignificant (advise equation: coefficient $=0.025, p=.612$; Quit equation: coefficient $=-0.004$, $p=.944)$. Therefore it is unlikely that our result was driven by the selection of health-minded patients to see physicians that practice more preventive care.

To see if different severity of observed conditions accounted for a significant part of our estimated effect of physician counseling, we estimate the model controlling for the individual diagnoses rather than the grouped 
conditions (see notes $\dagger$, $\ddagger$, and $\S$ of Table 1 for the list of diagnoses). Although by doing so we do not get at the severity differences that are "unobservable," if such an analysis yields similar results as in the original analysis where severity was less well controlled for, then unobserved severity differences of reported conditions should not be of great concern. This analysis indicated a 6.9 percent quitting rate under no advice, and 14.7 percent with advice, reflecting a 7.8 percentage point effect of some physician advice, identical with the results of the original model.

To assess possible biases because of all other unobserved factors including unobserved health conditions, we conducted a simulation exercise. The idea of the exercise is to generate simulated data where physician advice on smoking cessation had no effect, and then to explore conditions (and to evaluate the plausibility of the conditions) under which the IV method would yield misleading evidence that there is an effect. The exercise proceeds in four steps. In the first step, we start from the null hypothesis that the true treatment effect of physician advice on smoking cessation is zero (thereby setting the coefficient of smoking cessation advice in the quit equation to 0 ) and relax the exclusion restriction of the IVs. We vary the coefficient of the two IVs (assumed to be the same) in the quit equation from 0.0 (exclusion restriction holds) to 0.12 , with an increment of 0.02 . Meanwhile, we retain coefficients of all other covariates in the quit equation estimated from our original bivariate probit model. In the second step, we derive the predicted probability of quitting for each individual in the sample based on assumptions made in the first step. Based on the predicted probability of quitting, we generate a dichotomous quitting outcome for each individual using a random number generator. In the third step, we re-estimate the bivariate probit model using the simulated quit outcome generated in Step 2, excluding the IVs from the quit equation. We derive the incremental probability of quitting associated with the cessation advice (the "pseudo"-treatment effect estimated with the IV model, with the exclusion restriction wrongfully imposed). Because physician advice has no effect on quitting given how we construct the data, this pseudo-treatment effect represents an estimate of the bias introduced because of the exclusion of the IVs. For each value of the coefficient of the IVs (from 0.0 to 0.12 ) in the quit equation, we reiterate the analysis for 1,000 times and obtain the average estimated pseudo-treatment effect. We also derive the standard errors and 95 percent confidence intervals for the Monte Carlo precision of these point estimates, to reflect the uncertainty in the simulation.

Results of this analysis are presented in Table 4 . In the table, $\alpha_{\mathrm{IV}}$ stands for the simulated coefficient of the IVs in the quit equation, as our measure of 
Table 4: Results of Simulation to Check the Validity of the Exclusion Restriction

\begin{tabular}{cccc}
\hline$\alpha_{I V}$ & $\Delta P_{I V}$ & $r r_{I V}$ & Pseudo Treatment Effect and 95\% Confidence Interval \\
\hline 0.00 & 0.000 & 1.00 & $-0.003[-0.005,-0.001]$ \\
0.02 & 0.002 & 1.03 & $0.020[0.018,0.022]$ \\
0.04 & 0.005 & 1.07 & $0.041[0.039,0.043]$ \\
0.06 & 0.007 & 1.11 & $0.063[0.061,0.065]$ \\
0.08 & 0.009 & 1.14 & $0.084[0.082,0.086]$ \\
0.10 & 0.012 & 1.19 & $0.106[0.104,0.108]$ \\
0.12 & 0.015 & 1.23 & $0.128[0.126,0.130]$ \\
\hline
\end{tabular}

Notes: $\alpha_{\mathrm{IV}}$ is the simulated coefficient of the IVs in the outcome equation; $\Delta P_{\mathrm{IV}}$ is the incremental probability of quitting associated with $\alpha_{\mathrm{IV}} ; r_{\mathrm{IV}}$ is the relative risk of quitting associated with the IVs given $\alpha_{\mathrm{IV}}$. The last column lists the "treatment effect" of physician advice on smoking cessation estimated when the true effect is zero and the IVs are excluded from the outcome equation.

the degree of the violation of the exclusion restriction assumption; $\Delta P_{\text {IV }}$ stands for the incremental probability of quitting associated with the two IVs when $\alpha_{\mathrm{IV}}$ takes on the simulated value; $r r_{\mathrm{IV}}$ stands for the relative risk of quitting associated with the IVs given $\alpha_{\mathrm{IV}}$. The last column shows the pseudo-treatment effect averaged across the 1,000 runs. As shown in the table, when $\alpha_{\mathrm{IV}}$ was 0 (i.e., exclusion restriction holds), the pseudo-treatment effect was close to 0 , as expected. (It is not exactly zero because of the bias introduced by the nonlinear nature of the bivariate probit model.) Also as expected, the pseudotreatment effect increased with $\alpha_{\mathrm{IV}^{-}}$the greater the direct effect of the IVs on the outcome, the greater the bias from excluding them. Meanwhile, these data suggested that if our original estimate of the treatment effect (a 7.8 percentage point increase in the rate of quitting) were entirely biased because the IVs were inappropriately excluded, the IVs had to have a direct effect size of 1 percentage point on quitting and a relative risk of 1.14 , with the coefficient $\left(\alpha_{\mathrm{IV}}\right)$ being around 0.08 , which is comparable in magnitude to that of having serious cardiovascular conditions such as ever having a heart attack. ${ }^{6}$

Data presented in Sloan, Smith, and Taylor (2003, Table 6.4) based on the Health and Retirement Study suggested that the odds ratio of quitting over the past 2 years associated with the onset of a smoking-related health shock was at least three times as large as those associated with the onset of a nonsmoking health shock, onset of fair or poor health, onset of limitation in activities of daily living and death of a parent. ${ }^{7}$ Although we do not have data on the onset of health shocks given the cross-sectional nature of NHIS, data from the work of Sloan and colleagues do help shed some light on the plausibility of the conditions by which our IV estimate would be entirely biased. 
Given that the relative risk of quitting associated with ever having a diagnosis of serious cardiovascular condition in our study is 1.13 (authors' calculation based on the estimated model in Table 3), it is extremely unlikely that unobserved health conditions and other shocks would have a relative risk of that magnitude. Therefore it is unlikely that our result regarding the effect of physician advice was driven by unobserved factors such as unobserved health conditions.

\section{DISCUSSION}

Our analysis of the 2001 NHIS data suggests that physicians and other medical providers tended to give advice on smoking cessation to patients who were heavier smokers and/or patients who already had smoking-related conditions. Because of their smoking history, quitting was likely to be more difficult for this group. It is thus not surprising that patients advised to quit manifested worse cessation outcomes on average than those who did not receive any advice. Using IV analysis for provider advice in a joint model of smoking cessation outcome and provider advice, we found that some provider advice led to a 7.8 percentage point higher quit rate after the selection in provider advice was corrected for.

Previous RCTs (e.g., Russell et al. 1983; Jamrozik et al. 1984; Demers et al. 1990) found provider advice to increase 1-year abstinence modestly. For example, Demers and colleagues found 8 percent abstinence among the group that received 3-5 minutes unstructured advice versus 4 percent among the control; and the difference is not statistically significant. Jamrozik et al. (1984) found an increase from 11 to 15 percent for self-reported abstinence at 1 year due to receipt of verbal and written advice. Russell et al. (1983) reported 4.1 percent abstinence at 1 year for the group provided with advice and smokingcessation booklet, compared with 3.9 percent among the nonintervention control group.

The effect size of some provider advice (versus no advice at all) found in our study is larger than what were reported in these RCTs. First and foremost, the estimated effect in our study pertains to the combined effect of advice and possible additional help such as counseling, referral to cessation program and medication, which combined is likely to be more effective than pure advice. ${ }^{8}$ In addition, largely because of ethical reasons, either the control condition in RCTs was "routine care" (Demers et al. 1990), or studies allowed physicians to give advice to patients in the control arm (Stewart and Rosser 1982; Jamrozik 
et al. 1984) or stated that control patients should not receive any physician intervention/advice without reporting how such a condition was to be effectively enforced (Russell et al. 1979, 1983; Page et al. 1986; Slama et al. 1990). As a result

To put the estimated effect of provider advice into perspective, we collected data from various sources and computed the effects of major tobacco control strategies or policies in terms of the reduction in the number of smokers in the United States (Table 5). The data sources, assumptions made, and calculations conducted are summarized in the notes of Table 5 . As the exercise indicates, based on our results, if providers were to provide some advice for all of their smoking patients the way they are doing it now, compared with routine care, they would help almost 1.4 million more patients to quit during 1 year, an effect size that is twice as large as that of brief provider advice lasting for 3-5 minutes as tested in RCTs, and larger than that of doubling the current federal excise tax for cigarettes. Although more extensive state clean indoor air laws and banning smoking in private workplaces are associated with larger decreases in the number of smokers compared with some provider advice, in the longer run, one-time effects of legislation or policies are likely to be dwarfed by the effect of provider advice, which can be realized year after year.

Our study is subject to several limitations. First of all, our analyses were restricted to those who regularly visited their health care providers and therefore results do not generalize to the entire smoking population. ${ }^{9}$ However, because physician advice only takes effect when a patient sees a physician and since it is much more challenging to get patients who do not regularly see a provider to see a doctor, we believe that knowing the effect of physician advice among patients is of practical and policy significance. Second, one needs to be careful in extrapolating our IV results to the entire smoking population. While controlled trials answer the largely clinical question of the average efficacy of the treatment over a specified patient population, IV results are attributable to the effect among the subset of population who received different treatments because of the randomization based on the IVs (or the "marginal patients"; McClellan and Newhouse 2000). In our case, the "marginal patients" are those who were advised on smoking cessation because their providers advised them on diet and/or physical activity and carried the pattern to smoking cessation. While the IV estimates are informative of the effectiveness of incrementally increasing providers' smoking cessation advice, we do not know to what extent the results apply to people who are nonmarginal patients. This may have further given rise to the difference between our results 
2130 HSR: Health Services Research 41:6 (December 2006)

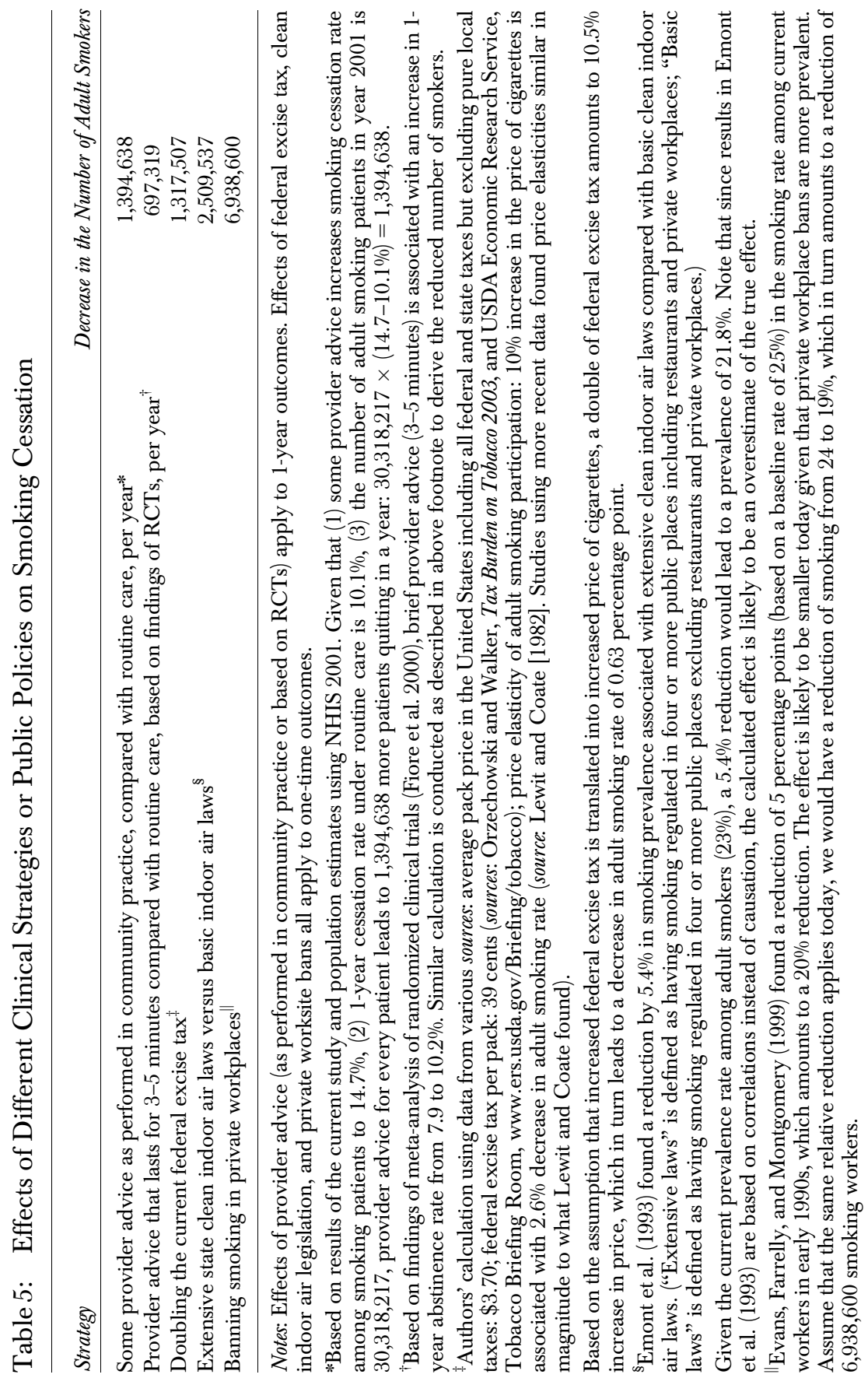


and what previous RCTs would imply regarding the effect of provider cessation advice.

Third, some data limitations and resulting caveats are worth mentioning. For smokers in our sample, the length of time between when treatment (advice) took place and when abstinence was measured may vary among patients. Therefore the estimated effect should not be interpreted as the outcome at exactly 1 year after the treatment. Our measure of the treatment (some provider advice on cessation) was based on patient self-report and therefore is subject to measurement error. However, there is evidence that patient perception of the content and quality of smoking cessation intervention right after their physician visits correlated well with audiotape assessment of the physician-patient interaction (Pbert et al. 1999). Other studies have shown that patient report of smoking cessation counseling based on exit survey had high sensitivity (0.7) and specificity (0.96) compared with direct observation (Stange et al. 1998). A more recent study tested the reliability of using patient survey data to measure patients' experience with physicians and found high intraphysician reliability of patient reports of smoking cessation counseling (Safran et al. 2004). Furthermore, since we use the variation in smoking cessation advice induced by variation in advice on diet/physical activity to identify the effect, the misreport of cessation advice should not impair the validity of our IV results unless some patients systematically misreport on both diet/ physical activity and smoking cessation advice.

Quitting smoking is not easy for most habitual smokers. Clinical protocols of smoking cessation intervention identified by RCTs are often not flexible enough to meet the needs of individual cases. Furthermore, they are often time and resource intensive and thus not feasible in routine clinical practices. Health providers in community practices, not knowing how effective they are in advising patients to quit smoking, are not advising their patients consistently. As a result, they forego opportunities of clinical intervention in smoking cessation especially for patients who are lighter smokers and/or who do not yet have smoking-related conditions. Yet it is for these patients that provider advice may actually make a bigger difference and the damages of tobacco consumption could potentially be avoided to a greater extent. Given the large effect we found for provider advice as practiced in routine care, health care providers should be encouraged and supported by their home organizations, managed care plans, and public policies, to deliver smoking cessation advice more broadly. 


\section{ACKNOWLEDGMENTS}

This research is funded by the National Cancer Institute. We thank three anonymous reviewers and the editor for their very thoughtful and helpful comments.

\section{NOTES}

1. With two IVs (provider advice for diet and for physical activity) and one endogenous treatment variable (provider advice for smoking cessation), the model is over-identified. We conducted the test of exclusion by including one of the IVs assuming that the other IV is excludable from the smoking cessation equation. Wald tests suggested that we could not reject the null that either IV is excludable $(p=.36$ for diet advice and $p=.24$ for PA advice). However, given that the two IVs are conceptually very similar and highly correlated (correlation coefficient: 0.63 ), it is not reasonable to assume that one is excludable and the other one not and therefore the validity of the test of exclusion is questionable in this case.

2. The unobserved conditions do not have to be related to either smoking or diet/ physical activity. Because smoking and diet/physical activity are important behaviors that affect health in general, a health shock of any kind may prompt a provider to advise against smoking and for healthy eating and physical activity; at the same time, patients may respond to such a shock by quitting smoking. Analysis based on the Health and Retirement Study (HRS) by Sloan, Smith and Taylor (2003) suggested that nonsmoking health shock, onset of limitations of activities, and onset of fair/poor health all predict quitting with statistical significance (Sloan, Smith and Taylor 2003; Chapter 6, Table 6.4).

3. It is not clear whether patients with unobserved health conditions related to smoking are more (or less) motivated to quit. On the one hand, quitting may become more pertinent with the onset of such conditions; on the other, people may become less motivated to quit given that they already incurred the damages of smoking to their health.

4. Arguably, some of the covariates, for example, indicator of the patient's working status and whether the patient lives with his/her spouse or partner, do not belong to the advice equation, since their role in affecting smoking cessation discussion is likely to be limited if not minimal. We include the full set of covariates in both equations to estimate an unconstrained model.

5. We focused on the privately insured and excluded publicly insured patients from this analysis because in some states, public insurance programs such as Medicare and Medicaid mandated enrollment in managed care plans, making the sample inappropriate for this purpose.

6. The IVs could be positively correlated with the unobserved conditions but as long as they are not perfectly correlated, the coefficient of the unobserved conditions, if we could observe them, would have to be greater than 0.08 . 
7. Although OR in general may not be a good proxy for relative risk (RR) (Zhang and Yu 1998), because the incidence of quitting smoking during the past 2 years was relatively low (about 15 percent as reported in Sloan, Smith, and Taylor [2003]), the deviation of OR from RR is of less concern. Besides, we are not able to derive RR based on the information provided in Sloan et al.

8. Results based on meta-analyses in Fiore et al. (2000) indicated that brief counseling lasting about 3 minutes was associated with an OR of 1.3 of quitting, and pharmacological aids were associated with an OR between 2 and 3. Therefore we believe that our estimated effect of some provider advice (with possible additional help such as prescription of pharmacological aids) is of reasonable size.

9. One of the reviewers suggested that we use health insurance as IVs to identify the effect of physician smoking cessation advice in an unconstrained population (patients and nonpatients), a strategy adopted in Kenkel and Terza (2001). However, unlike in that study where insurance seemed a plausible IV for physician advice to reduce alcohol consumption among moderate drinkers, in our case, the set of insurance variables were jointly significant $(p=.016)$ in predicting one's quitting outcome and thus could not be excluded from the quit equation. This is not surprising given that insurance coverage should facilitate access to pharmacological aids and intensive counseling, both of which are proven effective in helping patients quit.

\section{REFERENCES}

Angrist, J. 2001. "Estimation of Limited-Dependent Variable Models with Binary Endogenous Regressors: Simple Strategies for Empirical Practice.” Journal of Business and Economic Statistics 19 (1): 2-28.

Angrist, J., G. Imbens, and D. Rubin. 1996. "Identification of Causal Effects Using Instrumental Variables." Journal of the American Statistical Association 91: 444-55.

Assaf, A. R., D. Parker, K. L. Lapane, J. L. McKenney, and R. A. Carleton. 2002. “Are There Gender Differences in Self-Reported Smoking Practices? Correlation with Thiocyanate and Cotinine Levels in Smokers and Nonsmokers from the Pawtucket Heart Health Program.” Journal of Womens Health (Larchmt) 11 (10): 899906.

Bhattacharya, J., D. Goldman, and D. McCaffrey. 2006. "Estimating Probit Models with Self-Selected Treatments." Statistics in Medicine 25 (3): 389-413.

Demers, R. Y., A. V. Neale, R. Adams, C. Trembath, and S. C. Herman. 1990. "The Impact of Physicians' Brief Smoking Cessation Counseling: A MIRNET Study.” Journal of Family Practice 31 (6): 625-9.

Emont, S. L., W. S. Choi, T. E. Novotny, and G. A. Giovino. 1993. "Clean Indoor Air Legislation, Taxation, and Smoking Behavior in the United States: An Ecological Analysis." Tobacco Control 2: 13-17.

Evans, W. N., M. C. Farrelly, and E. Montgomery. 1999. "Do Workplace Smoking Bans Reduce Smoking?” American Economic Review 89 (4): 728-47. 
Fiore, M. C., W. C. Baily, S. J. Cohen, S. F. Dorfman, M. G. Goldstein, E. R. Gritz, R. B. Heyman, C. R. Jaén, T. E. Kottke, H. A. Lando, R. E. Mecklenburg, P. Dolan Mullen, L. M. Nett, L. Robinson, M. L. Stitzer, A. C. Tommasello, L. Villejo, and M. Wewers. 2000. Treating Tobacco Use and Dependence. Clinical Practice Guideline. Rockville, MD: U.S. Department of Health and Human Services.

Fiore, M. C., T. E. Novotny, J. P. Pierce, G. A. Giovino, E. J. Hatziandreu, P. A. Newcomb, T. S. Surawicz, and R. M. Davis. 1990. "Methods Used to Quit Smoking in the United States. Do Cessation Programs Help?" Journal of the American Medical Association 263 (20): 2760-5.

Gilpin, E. A., J. P. Pierce, M. Johnson, and D. Bal. 1993. "Physician Advice to Quit Smoking: Results from the 1990 California Tobacco Survey." Journal of General Internal Medicine 8 (10): 549-53.

Greene, W. H. 2000. Econometric Analysis. Upper Saddle River, NJ: Prentice-Hall.

Jaén, C. R., K. C. Stange, L. M. Tumiel, and P. Nutting. 1997. "Missed Opportunities for Prevention: Smoking Cessation Counseling and the Competing Demands of Practice." Journal of Family Practice 45 (4): 348-54.

Jamrozik, K., M. Vessey, G. Fowler, N. Wald, G. Parker, and H. Van Vunakis. 1984. "Controlled Trial of Three Different Antismoking Interventions in General Practice." British Medical Journal (Clinical Research Edition) 288 (6429): 1499-503.

Kenkel, D. S., and J. V. Terza. 2001. "The Effect of Physician Advice on Alcohol Consumption: Count Regression with an Endogenous Treatment Effect.” Journal of Applied Econometrics 16: 165-84.

Kottke, T. E., R. N. Battista, G. H. DeFriese, and M. L. Brekke. 1988. “Attributes of Successful Smoking Cessation Interventions in Medical Practice. A Meta-Analysis of 39 Controlled Trials." Journal of the American Medical Association 259 (19): 2883-9.

Lewit, E. M., and D. Coate. 1982. "The Potential for Using Excise Taxes to Reduce Smoking." Journal of Health Economics 1 (2): 121-45.

McClellan, M. B., and J. P. Newhouse. 2000. "Overview of the Special Supplement Issue." Health Service Research 35 (5 Part 2): 1061-9.

National Center for Health Statistics. 2003a. Health, United States, 2003. Hyattsville, MD: National Center for Health Statistics.

2003b. National Health Interview Survey: Description.” [accessed on August 10, 2004]. Available at http://www.cdc.gov/nchs/about/major/nhis/hisdesc.htm

Page, A. R., D. J. Walters, R. P. Schlegel, and J. A. Best. 1986. "Smoking Cessation in Family Practice: The Effects of Advice and Nicotine Chewing Gum Prescription." Addictive Behavior 11 (4): 443-6.

Patrick, D. L., A. Cheadle, D. C. Thompson, P. Diehr, T. Koepsell, and S. Kinne. 1994. "The Validity of Self-reported Smoking: A Review and Meta-analysis." American Journal of Public Health 84 (7): 1086-93.

Pbert, L., A. Adams, M. Quirk, J. R. Hebert, J. K. Ockene, and R. S. Luippold. 1999. "The Patient Exit Interview as an Assessment of Physician-Delivered Smoking Intervention: A Validation Study." Health Psychology 18 (2): 183-8. 
Pokorski, T. L., W. W. Chen, and R. L. Bertholf. 1994. "Use of Urine Cotinine to Validate Smoking Self-Reports in U.S. Navy Recruits." Addictive Behaviors 19 (4): 451-4.

Russell, M. A., R. Merriman, J. Stapleton, and W. Taylor. 1983. "Effect of Nicotine Chewing Gum as an Adjunct to General Practitioner's Advice against Smoking." British Medical Journal (Clinical Research Edition) 287 (6407): 1782-5.

Russell, M. A., C. Wilson, C. Taylor, and C. D. Baker. "Effect of General Practitioners' Advice against Smoing." British Medical Journal 2: 231-5.

Safran, D., M. Karp, K. Coltin, J. Ogren, A. Li, H. Chang, and W. Rogers. 2004. "Measuring Patients' Experiences with Individual Physicians. Abstract Accepted for the 27th Society of General Internal Medicine Annual Meeting (Chicago, IL).” Journal of General Internal Medicine 19 (suppl 1): 109.

Slama, K., S. Redman, J. Perkins, A. L. Reid, and R. W. Sanson-Fisher. 1990. "The Effectiveness of Two Smoking Cessation Programmes for Use in General Practice: A Randomised Clinical Trial." British Medical Journal 300: 1707-9.

Sloan, F. A., V. K. Smith, and D. H. Taylor. 2003. The Smoking Puzzle: Information, Risk Perception, and Choice. Cambridge, MA: Harvard University Press.

Stange, K. C., S. J. Zyzanski, T. F. Smith, R. Kelly, D. M. Langa, S. A. Flocke, and C. R. Jaén. 1998. "How Valid Are Medical Records and Patient Questionnaires for Physician Profiling and Health Services Research? A Comparison with Direct Observation of Patients Visits." Medical Care 36 (6): 851-67.

Stewart, P. J., and W. W. Rosser. 1982. "The Impact of Routine Advice on Smoking Cessation from Family Physicians.” Candian Medical Association Journal 126 (9): 1051-4.

Thorndike, A. N., N. A. Rigotti, R. S. Stafford, and D. E. Singer. 1998. "National Patterns in the Treatment of Smokers by Physicians." Journal of the American Medical Association 279 (8): 604-8.

Zhang, J., and K. F. Yu. 1998. "What's the Relative Risk? A Method of Correcting the Odds Ratio in Cohort Studies of Common Outcomes." Journal of the American Medical Association 280 (19): 1690-1. 